\title{
REVERSING THE SUPERNOVA ORDER IN MASSIVE BINARIES
}

\author{
ONNO R. POLS \\ Astronomical Institute 'Anton Pannekoek', University of Amsterdam \\ Kruislaan 403, 1098 SJ Amsterdam, The Netherlands \\ Centre for High Energy Astrophysics, Amsterdam \\ Institute for Theoretical Physics, University of California, Santa Barbara
}

\begin{abstract}
Using simultaneous evolutionary calculations of both components of a massive binary, it is shown that the initial secondary component can explode before the primary, if Roche lobe overflow occurs on the main sequence. Also, it is found that initially wide binaries are more likely to come into contact than close ones.
\end{abstract}

\section{Introduction}

During the past 25 years, stellar evolution codes have been widely used to study the evolution of close binaries. However, in most cases only the mass-losing (primary) star is well-studied and the mass-gaining (secondary) component receives relatively little attention. Usually, simplifying 'conservative' assumptions are made: the mass gainer is inert and accepts all the mass that is transferred to it, so that total mass and angular momentum are conserved.

Ulrich and Burger (1976) and others have shown that the reaction of the secondary to accretion is a rapid expansion, if the mass ratio of the binary is very different from unity. As a result, a contact or common-envelope binary is formed and more or less extensive mass and angular momentum losses may be expected (Flannery and Ulrich, 1977). Especially in case A binaries, where the mass loser is a main sequence star, contact is usually assumed to be inevitable because of the initial closeness of the components. Some papers have investigated the simultaneous evolution of both components of case A systems, up to and partly through the contact stage (e.g. Webbink, 1976). The possibility that some case A binaries might avoid contact, and the subsequent evolution of such systems, have been little investigated.

An intriguing possibility for massive case A systems that avoid contact and evolve conservatively is that the order in which the components explode as supernovae can be reversed, as we will show in section 2 . This is compared to the situation for case B systems. In section 3 the results are discussed.

\section{Evolutionary calculations}

The evolution of both stars of a close binary system is calculated simultaneously, including the mass transfer phases, using the Eggleton stellar evolution code (Eggleton, 1971, 1972, 1973). During the mass transfer, conservation of total mass and angular momentum is 
assumed as long as the system is semi-detached. The reaction of the secondary to accretion is taken into account, assuming that the accreted matter falls gently onto the surface (i.e., the potential energy is radiated away) and that it has the same specific entropy as the surface layers of the mass gainer. Neither overshooting nor stellar wind mass loss is included.

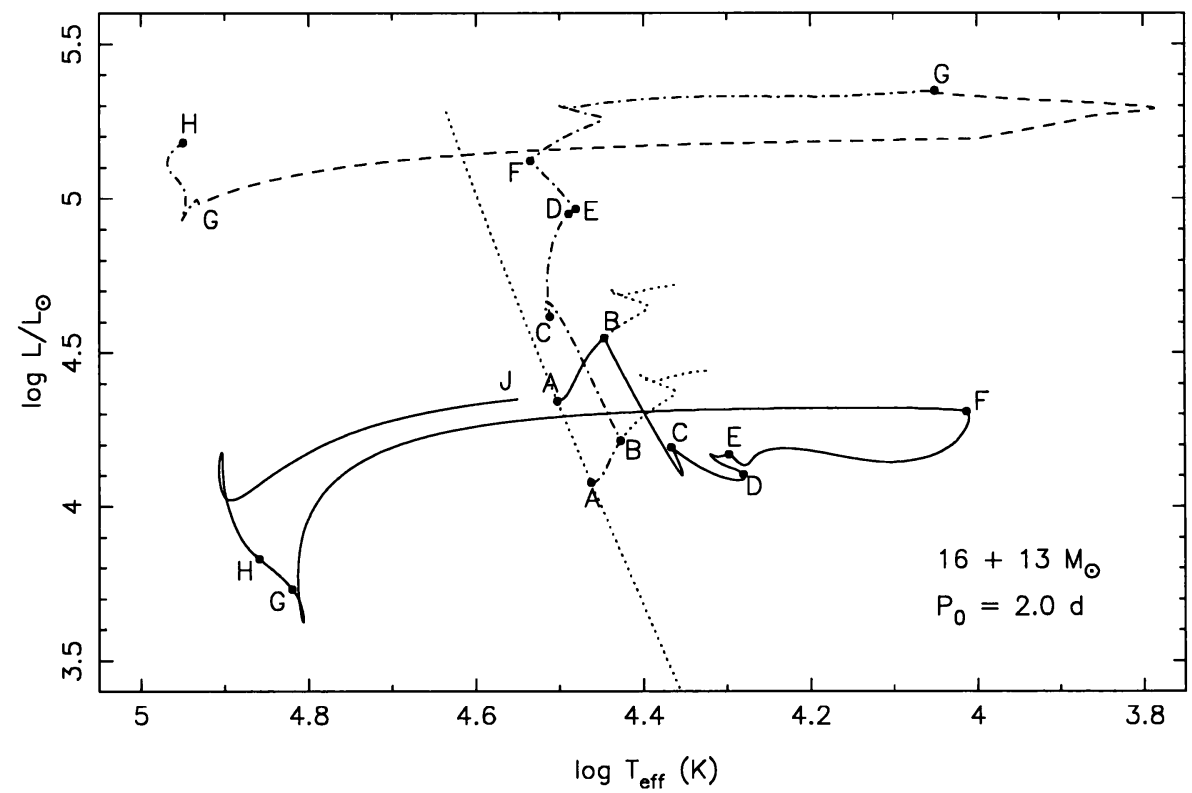

Figure 1. Evolutionary tracks in the HR diagram of a $16 M_{\odot}$ (solid line) $+13 M_{\odot}$ (dashdot) case A binary. The lettering along the curves corresponds to that in Table 1 . The dashed curve indicates the 'stripping' of the secondary, the dotted curves are the ZAMS and the tracks of single $16 M_{\odot}$ and $13 M_{\odot}$ models.

Table 1. Evolutionary time scales

\begin{tabular}{l|c|c|r|r|l|l}
\hline & $t\left(10^{6} \mathrm{yr}\right)$ & $P_{\text {orb }}(\mathrm{d})$ & $M_{1}$ & $M_{2}$ & description & $\dot{M}_{\max }\left(M_{\odot} / \mathrm{yr}\right)$ \\
\hline $\mathrm{A}$ & 0.000 & 1.97 & 16.0 & 13.0 & ZAMS & \\
$\mathrm{B}$ & 7.700 & 1.97 & 16.0 & 13.0 & start $1^{\text {st }}$ mass transfer & $3.410^{-4}$ \\
$\mathrm{C}$ & 7.770 & 2.45 & 10.4 & 18.6 & start $2^{\text {nd }}$ mass transfer & $1.310^{-6}$ \\
$\mathrm{D}$ & 12.213 & 4.72 & 7.1 & 21.9 & end $2^{\text {nd }}$ mass transfer & \\
$\mathrm{E}$ & 12.445 & 4.72 & 7.1 & 21.9 & start $3^{\text {rd }}$ mass transfer & $7.410^{-5}$ \\
$\mathrm{~F}$ & 12.570 & 73.8 & 2.3 & 26.7 & end $3^{\text {rd }}$ mass transfer & \\
G & 14.712 & 73.8 & 2.3 & 26.7 & start spiral in & $>10^{-1}$ \\
G' & $14.72:$ & $?$ & 2.3 & 7.5 & end spiral in & \\
H & 15.402 & $?$ & 2.3 & 7.5 & explosion secondary & \\
J & 15.694 & - & 2.3 & 1.4 & explosion primary &
\end{tabular}




\subsection{EVOLUTION OF THE CASE A SYSTEM}

In Fig. 1 the evolution of a system consisting of a $16 M_{\odot}$ and a $13 M_{\odot}$ star, with an initial orbital period of 2 days, is shown in the HR diagram. Three phases of mass transfer from the primary to the secondary can be distinguished (see Table 1). During the first, rapid phase the secondary expands somewhat out of thermal equilibrium, but contact is avoided. The mass transfer rate reaches a minimum at point $\mathrm{C}$, but then mass transfer continues in a long semi-detached (Algol) phase on a nuclear timescale, until the primary contracts when its central hydrogen is exhausted. After a short detached phase the primary again fills its Roche lobe and a third, thermal-timescale phase ensues, terminated when helium ignites in the primary which then contracts towards the helium main sequence.

The secondary, which is rejuvenated during the mass transfer phases, subsequently fills its Roche lobe (point G). However, because of the extreme mass ratio a very high mass transfer rate is attained which cannot be followed with the code. Presumably, the helium star spirals into the secondary's envelope. We have modelled this phase by artificially stripping the secondary of its envelope until its radius becomes very small, while leaving the primary unaltered (i.e. assuming that it does not accrete or lose mass during the spiral-in).

A double helium star in a very close orbit is the most likely outcome of the spiral-in. The orbital period is difficult to estimate, presumably it will be less than a day. Both stars will undergo supernova explosions (the lower mass limit for a helium star to explode being about $2.2 M_{\odot}$, Habets 1986 ), the secondary remnant about $310^{5}$ years before the primary.

\subsection{COMPARISON WITH CASE B SYSTEMS}

We have also computed the evolution of a case B system with the same masses but with an orbital period of 6 days. After one rapid phase of mass transfer, a $3.7 M_{\odot}$ helium star is formed which explodes $3.110^{6}$ years before the $25.3 M_{\odot}$ companion fills its Roche lobe. Apparently, it is principally the mass of the helium star that determines which component explodes first. The masses and evolutionary time scales of the secondaries are not too different in either case. However, in the case A system the formation of a massive helium star is prevented because the size of the convective core has decreased as a result of mass transfer.

A remarkable feature is that the case B system came closer to contact than the case A system. Because the peak mass transfer rate is about six times as large, the secondary expanded much more and almost touched its Roche lobe. Calculations of series of case B models of smaller masses $\left(8+4 M_{\odot}\right)$ and increasing orbital period confirm that wide binaries more easily evolve into contact because of higher mass transfer rates. This is contrary to the common idea that closer systems are more likely to come into contact.

\section{Discussion}

The failure of case B systems to reverse the supernova order was already found by Hellings (1984). Even for mass ratios close to 1 , the secondary is always so much rejuvenated that its evolution is retarded. Only if the mass ratio is almost 1 , so that the secondary has already left the main sequence when mass transfer starts, it will not be rejuvenated and it is possible that it explodes before the primary (P. Podsiadlowski, private communication). We will further investigate this possibility. 
Our case A system will most probably unbind during the first supernova explosion, because more than half of the system mass is blown away if a $1.4 M_{\odot}$ neutron star is formed. Hence, a runaway pulsar and a runaway helium star are formed. This scenario may apply to the Crab nebula, which was formed about 200 pc above the Galactic plane and for which there is some evidence that the progenitor was a helium star.

We conclude by discussing how the assumptions made in the calculation might influence the results. We neglected the possibility that the mass gainer may be spun up by the accretion which could facilitate the loss of mass and angular momentum (Packet, 1981). On the other hand, it is possible that the accreted matter has much lower specific entropy than the stellar surface which would make contact systems less likely (Shu and Lubow, 1981). Convective overshooting or stellar wind mass loss will increase the time scales involved, both of the helium star phase and of the main sequence phase. The precise effect thus depends strongly on which of these is affected the most. This requires further investigation.

Acknowledgements. It is a pleasure to thank Peter Eggleton, Robert Cannon and Dipankar Bhattacharya for useful discussions and suggestions. This work was supported in part by the National Science Foundation under Grant no. PHY89-04035.

\section{References}

Eggleton, P.P. (1971) M.N.R.A.S. 151, 351

Eggleton, P.P. (1972) M.N.R.A.S. 156, 361

Eggleton, P.P. (1973) M.N.R.A.S. 163, 279

Flannery, B.P., Ulrich R.K. (1977) Ap. J. 212, 533

Habets, G.M.H.J. (1986) Astron. Ap. 167, 91

Hellings, P. (1984) Ap. Space Sci. 104, 83

Packet, W. (1981) Astron. Ap. 102, 17

Shu, F.H., Lubow, S.H. (1981) Ann. Rev. Astron. Ap. 19, 277

Ulrich, R.K., Burger H.L. (1976) Ap. J. 206, 509

Webbink, R.F. (1976) Ap. J. Suppl. 32, 583 